Raquel Santos Robalo Fernandes ${ }^{1}$

Ana FILIPA BERNARDINo SIMÕes'

ana Cristina Neves Figueiredo

Ana Rita Silva Ribeiro ${ }^{2}$

Francisca Maria Ferreira Aleixo ${ }^{3}$

Sillvia Margarida Duarte Texera

GuerRa Aragǘs ${ }^{3}$

Nilla Yakalage Barreira Amaral ${ }^{3}$

\section{Prognóstico obstétrico de pacientes portadoras de diabetes mellitus pré-gestacional}

\author{
Pregnancy outcomes in women with pre-existing diabetes
}

Artigo Original

Palavras-chave

Diabetes mellitus/metabolismo Diabetes gestacional/metabolismo Complicações na gravidez Resultado da gravidez

Keywords

Diabetes melittus/metabolism Diabetes, gestational/metabolism Pregnancy complications Pregnancy outcome

\section{Resumo}

OBJETIVO: Avaliar as alterações epidemiológicas, de perfil clínico e de prognóstico obstétrico em pacientes portadoras de diabetes mellitus pré-gestacional. MÉTODOS: Estudo retrospetivo (coorte) de todas as gestações simples, com diagnóstico de diabetes prévio que foram seguidas num centro com apoio perinatal diferenciado entre 2004 e 2011 (n=194). Analisaram-se tendências relacionadas com dados demográficos e variáveis clínicas maternas, dados de indicadores de cuidados pre-concepcionais e durante a gravidez, e de controle metabólico. Dados do parto como a idade gestacional $(\mid G)$ do parto, via do parto e peso do neonato foram variáveis também estudadas. RESULTADOS: A frequência global de diabetes prévia, durante o período estudado, foi de 4,4 por mil, não se verificando variações significativas durante o período de estudo. Os casos de diabetes tipo 2 permaneceram constantes. Em $67 \%$ dos casos o parto foi de termo (máximo de 80\% em 2010-201 1), registrou-se uma redução significativa dos partos por cesárea eletiva $(p=0,03$ ) e na incidência de neonatos considerados grandes para a IG $(p=0,04)$ ao longo dos anos em estudo. Apesar dos bons resultados relacionados com o controle metabólico ao longo da vigilância da gravidez não foi registrada nenhuma melhora ao longo do tempo. Da mesma forma a proporção de gestantes diabéticas com avaliação pre-concepcional permaneceu pouco animadora. CONCLUSÕES: $\bigcirc$ seguimento de gestantes portadoras de diabetes mellitus em unidades multidisciplinares parece permitir um ajuste metabólico tão precoce quanto possível, de forma a conseguir melhorar o prognóstico obstétrico. A melhora nos cuidados pré-concepcionais continua sendo um desafio.

\section{Abstract}

PURPOSE: To describe trends in prevalence, indicators of care and pregnancy outcomes for women with pre-existing type I or type || diabetes. METHODS: Cohort study of all consecutive singleton pregnancies complicated by pre-existing type | or type II diabetes followed from 2004 to 2011 at a tertiary perinatal care centre ( $n=194)$. We collected data from the medical records and described trends in demographics, clinical history, indicators of care before or during pregnancy and glycaemic control. We also studied perinatal outcomes, including gestational age at delivery, mode of delivery, and birthweight. RESULTS: The overall incidence of pregestational diabetes was 4.4 per 1000, with no significant changes throughout the study period. The number of type 2 diabetes cases also remained constant. In $67 \%$ of cases delivery occurred after 37 weeks (maximum 80\% in 2010-11). During this period there was a significant reduction in rates of elective caesarean section ( $p=0.03$ ) and in the incidence of large infants for gestational age ( $p=0.04)$. Indicators of glycaemic control were favorable throughout pregnancy, with no significant trends detected during the study period. However, preconceptional care indicators were substandard, with no significant improvement. CONCLUSIONS: A multidisciplinary approach to diabetic management and obstetric practice contributed to adequate glycaemic control throughout pregnancy and to improved pregnancy outcomes. Preconceptional care remains a key challenge.
Correspondênci

Raquel Robalo

Servico de Medicina Materno-Fetol Maternidade Dr. Alfredo da Costo

Rua Virioto

CEP: 1069-089

Lisboa, Portugal

Recebido

20/06/2012

Aceito com modificacōos

$15 / 10 / 2012$
Trabalho realizado no Serviço de Medicina Materno-Fetal da Maternidade Dr. Alfredo da Costa - Lisboa, Portugal.

'Serviço de Ginecologia e Óbstetrícia, Maternidade Dr. Alfredo da Costa - Lisboa, Portugal.

${ }^{2}$ Serviço de Ginecologia e Obstetrícia, Hospital de Santarém, EPE (Santarém) - Portugal.

${ }_{3}^{3}$ Maternidade Dr. Alfredo da Costa - Lisboa, Portugal. 


\section{Introdução}

A prevalência em mulheres em idade reprodutiva vem aumentando. Um estudo retrospetivo de 175.249 gestantes demonstrou que a frequência — que era de $0,8 \%$ em 1999 - aumentou para 1,8\% em $2005^{1}$. O diabetes preexistente à gravidez está associado aos piores resultados obstétricos e perinatais ${ }^{2,3}$. Com o intuito de melhorar o prognóstico obstétrico, a importância da avaliação pré-concepcional de mulheres com DM tipo 1 ou tipo 2 não deve ser negligenciada. O mau controle metabólico no período da organogênese associa-se a um aumento da taxa de aborto espontâneo e de malformações fetais ${ }^{4,5}$. Durante o tempo de gestação subsequente aumenta o risco de macrossomia e das suas comorbidades associadas. Também contribuem para os maus resultados obstétricos as complicações relacionadas com a doença, como a vasculopatia, o aumento do risco de parto pré-termo, restrição do crescimento fetal, doença hipertensiva da gravidez e morbimortalidade perinatal ${ }^{6}$.

Estudos recentes, de base populacional, revelaram que nas últimas duas décadas, apesar do esforço de melhoras nos cuidados da população de gestantes diabéticas, estas continuaram apresentando os piores resultados obstétricos e perinatais, quando comparadas com gestantes não diabéticas, e que as taxas de mortalidade perinatal, malformações fetais e macrossomia não sofreram melhoras significativas ${ }^{7,8}$.

Tendo como objetivo melhorar o prognóstico obstétrico de mulheres com diagnóstico de DM prévio à gravidez, é fundamental apostar na formação de equipes multidisciplinares com obstetras, endocrinologistas, nutricionistas e enfermeiros empenhados na otimização do controle metabólico da grávida diabética, com experiência na terapêutica hipoglicemiante adaptada às novas necessidades na gravidez, no incentivo constante do cumprimento da dieta e na promoção do exercício físico. A melhor interligação com os cuidados de saúde primários e associações de diabéticos tem como meta aumentar o número de gestações planejadas e, consequentemente, melhorar o controle metabólico dessas mulheres?.

O objetivo deste estudo é avaliar as alterações epidemiológicas, de perfil clínico e de prognóstico obstétrico em pacientes portadoras de DM pré-gestacional na Região Central de Portugal.

\section{Métodos}

Foi efetuado um estudo retrospetivo, de coorte, que incluiu todas as gestações simples, com diagnóstico de diabetes prévio, com vigilância na Maternidade Dr. Alfredo da Costa, em Lisboa, entre $1^{\circ}$ de janeiro de 2004 e 31 de dezembro de 2011 ( $n=194)$.

Definiu-se diabetes prévia como DM tipo 1 ou tipo 2, diagnosticada até pelo menos um ano antes da data prevista do parto. Foram excluídas gestantes que optaram pela interrupção voluntária da gravidez, em que o parto não ocorreu na nossa instituição e casos sem dados maternos ou perinatais completos.

Com as consultas dos prontuários obtivemos dados da história clínica, indicadores de cuidados pré-natais e de controle metabólico na gestação, e informação relativa ao parto. Foram avaliadas variáveis como a idade materna, tipo de DM, paridade, raça e índice de massa corporal (IMC) materno. Foram analisados os dados de cuidados pré-concepcionais e gestacionais, destacando-se a realização de consulta pré-concepcional e a idade gestacional (IG), em semanas completas, na entrada da consulta (determinada por datação no $1^{\circ}$ trimestre). $\mathrm{O}$ ganho ponderal durante a gestação foi definido como insuficiente, adequado ou excessivo de acordo com a IG do parto, e o IMC pré-gestacional de acordo com as guidelines do Institute of Medicine ${ }^{10}$.

O controle metabólico foi avaliado na data de entrada no seguimento de CGD e pelo menos uma vez por trimestre através do valor de hemoglobina glicosilada (HbA1c), definindo-se como bom controle metabólico quando esse valor se encontrava abaixo de $6,5 \%{ }^{11}$.

Foram analisadas as variáveis do parto: IG, via de parto e peso do recém-nascido $(\mathrm{RN})$. Considerou-se gravidez de termo quando IG foi superior a 37 semanas completas. Considerou-se aborto as situações de perda fetal antes da $24^{a}$ semana de gestação.

A macrossomia fetal foi definida para um peso ao nascer superior a $4000 \mathrm{~g}$. Os casos em que o peso ao nascimento era superior ao percentil 90 do peso para a idade gestacional e gênero ${ }^{12}$ foram definidos como recém-nascidos grandes para a idade gestacional (GIG).

$\mathrm{Na}$ análise das diferentes tendências ao longo dos anos em estudo agrupou-se a amostra em quatro grupos, com igual período de tempo (2004-2005, 2006-2007, 2008-2009 e 2010-2011). Testou-se a associação entre a evolução temporal em grupos e os diferentes parâmetros em estudo por meio da análise de variância (one-way ANOVA) para variáveis contínuas com distribuição normal, e por meio do teste do $\chi^{2}$ (associação e tendência) para variáveis categoriais. A análise estatística foi realizada recorrendo-se ao programa SPSS, versão 16 para Windows, e o nível de significância adotado foi $\mathrm{p}<0,05$.

Pelas características da metodologia utilizada, a apreciação pelo Comitê de Etica Médica e a necessidade de obtenção de termo de consentimento livre e esclarecido foram dispensadas como prevê a legislação portuguesa.

\section{Resultados}

No estudo foram incluídas 194 gestantes com diagnóstico de DM tipo 1 ou tipo 2, seguidas na nossa instituição entre 2004 e 2011. Registrou-se o nascimento de 168 recém-nascidos (81\%), 20 casos de aborto espontâneo (90\% no primeiro 
trimestre) e 6 casos de interrupção médica da gravidez por opção do casal (um caso de síndrome malformativo e cinco casos de trissomia 21), todos antes da $24^{a}$ semana de gravidez, após deferimento da Comissão Técnica de Certificação e dentro do enquadramento legal em vigor em Portugal.

Num universo de 44593 partos a incidência de diabetes prévia, durante os 8 anos estudados, foi de $4.4 \%$. Cento e vinte e quatro gestantes (64\%) tinham DM tipo 1 e $70(36 \%)$ eram gestantes com DM tipo 2. O número de casos com diabetes tipo 2 permaneceu constante.

Em relação à raça, 36\% das gestantes com DM tipo 2 eram de raça não branca versus $11 \%$ das gestantes com DM tipo $1(\mathrm{p}<0,001)$. Outras características maternas e sua variação ao longo do período em estudo podem ser consultadas na Tabela 1. Ao longo dos anos houve uma redução da proporção de gravidez em diabéticas nulíparas desde 2006-2007.

Constatou-se que a média de idade materna e a proporção de gestantes com idade superior a 35 anos, nos anos 2008-2009 foi significativamente mais elevada quando comparada com os anos restantes, dentro do período estudado $(\mathrm{p}=0,02)$. Apesar de não ter havido, observou-se, ao longo dos anos, uma diminuição dos casos de gestantes com excesso de peso ou obesas.

Vários indicadores de cuidados pré-natais foram avaliados, como a existência de consulta pré-concepcional, o início da vigilância hospitalar até à décima semana de gestação ou a avaliação de controle metabólico no primeiro trimestre de gravidez. Apenas 15,5\% do total dos casos havia tido uma consulta pré-concepcional. Em $64 \%$ dos casos a primeira consulta foi efetuada até a $10^{\mathrm{a}}$ semana de gestação; em $77 \%$ foi possível obter uma avaliação do valor de $\mathrm{HgA} 1$ c até a $12^{a}$ semana e em $95 \%$ dos casos foi feita a avaliação do fundo ocular no $1^{\circ}$ trimestre. Essas variáveis mantiveram-se estáveis ao longo do período estudado.

Tabela 1. Características maternas e variacão dos parâmetros ao longo dos anos (2004-2011)

\begin{tabular}{|c|c|c|c|c|c|}
\hline & $\begin{array}{c}2004- \\
2005 \\
(n=47)\end{array}$ & $\begin{array}{c}2006- \\
2007 \\
(n=45)\end{array}$ & $\begin{array}{c}2008- \\
2009 \\
(n=55)\end{array}$ & $\begin{array}{c}2010- \\
2011 \\
(n=47)\end{array}$ & $\begin{array}{c}\text { Valor } \\
\mathbf{p}\end{array}$ \\
\hline $\begin{array}{l}\text { Incidência } \\
\text { (por } 1000 \text { partos) }\end{array}$ & 4,1 & 3,9 & 5 & 4,3 & - \\
\hline $\begin{array}{l}\text { Idade materna } \\
\text { média ( }\left( \pm \mathrm{DP}^{\prime}\right)\end{array}$ & $31(6)$ & $31(6)$ & $33(6)$ & $31(6)$ & 0,1 \\
\hline $\begin{array}{l}\text { Idade materna >35 } \\
\text { A: } n(\%)\end{array}$ & $13(27,7)$ & $12(26,7)$ & $21(38,2)$ & $15(31,9)$ & 0,5 \\
\hline Etnia não branca: n (\%) & $7(14,9)$ & $10(22,2)$ & $11(20)$ & $11(23,4)$ & 0,7 \\
\hline Nuliparidade: n (\%) & $24(51,1)$ & $31(68,9)$ & $32(58,2)$ & $26(55,3)$ & 0,3 \\
\hline $\begin{array}{l}\text { Excesso de peso e } \\
\text { obesidade: n (\%) }\end{array}$ & $23(50)$ & $23(53,5)$ & $27(49,1)$ & $17(37,8)$ & 0,4 \\
\hline Diabetes tipo 2: n (\%) & $19(40,4)$ & $17(37,8)$ & $17(30,9)$ & $17(36,2)$ & 0,7 \\
\hline
\end{tabular}

*DP: desvio padrão.
Entre os casos em que ocorreu perda fetal antes da $24^{\mathrm{a}}$ semana verificou-se que o valor de $\mathrm{HgA} 1 \mathrm{C}$ média no $1^{\circ}$ trimestre foi superior, quando comparado com as gestantes que não tiveram perda fetal em igual período $(8,7$ versus $7,1 ; \mathrm{p}=0,001)$. Verificou-se, também, que a proporção de casos cujo o valor de $\mathrm{HgA1C}$ foi superior a $6,5 \%$ foi significativamente mais elevada entre as gestantes que experimentaram perda fetal antes da $24^{\mathrm{a}}$ semana, quando comparadas com as restantes ( $15 \%$ versus $2 \% ; \mathrm{p}=0,01)$.

Indicadores de cuidados pré-natais (Tabela 2) e os dados do parto (Tabela 3) foram estudados após exclusão dos casos de perda gestacional antes da $24^{\mathrm{a}}$ semana $(\mathrm{n}=168)$.

Valores de bom controle metabólico (definido como valor de $\mathrm{HgA} 1 \mathrm{c}$ inferior a 6,5\%) foram mais prevalentes no segundo e terceiro trimestres de gravidez, não tendo sido registradas melhoras ao longo dos anos em estudo.

Não foi registrada nenhuma alteração, com significância, nos dados relativos ao ganho ponderal das gestantes, ao longo dos anos de estudo. Porém, destacam-se os valores de ganho ponderal excessivo em 39\% dos casos. Em 42 gestantes com valores de IMC prévio superior $25 \%$ o ganho

Tabela 2. Indicadores de cuidados pré-natais nos casos de parto após as 24 semanas

\begin{tabular}{|c|c|c|c|c|c|}
\hline & $\begin{array}{c}2004- \\
2005 \\
(n=46) \\
\end{array}$ & $\begin{array}{c}2006- \\
2007 \\
(n=40)\end{array}$ & $\begin{array}{c}2008- \\
2009 \\
(n=42)\end{array}$ & $\begin{array}{c}2010- \\
2011 \\
(n=40)\end{array}$ & Valor $p$ \\
\hline \multicolumn{6}{|c|}{ Indicadores de cuidados durante a gravidez } \\
\hline $\begin{array}{l}\text { Ecocardiograma } \\
\text { fetal-18-22 } \\
\text { semanas: } n(\%)\end{array}$ & $38(82,6)$ & $35(87,5)$ & $38(90,5)$ & $36(90)$ & 0,6 \\
\hline $\begin{array}{l}\text { Aumento ponderal } \\
\text { insuficiente: } n(\%)\end{array}$ & $8(17,8)$ & $7(18,4)$ & $8(19)$ & $13(32,5)$ & \\
\hline $\begin{array}{l}\text { Aumento ponderal } \\
\text { adequado: n (\%) }\end{array}$ & $19(42,2)$ & $15(39,5)$ & $18(42,9)$ & $11(27,5)$ & 0,6 \\
\hline $\begin{array}{l}\text { Aumento ponderal } \\
\text { excessivo: n (\%) }\end{array}$ & $18(40)$ & $16(42,1)$ & $16(38,1)$ & $16(40)$ & \\
\hline \multicolumn{6}{|c|}{ Indicadores de controle metabólico* } \\
\hline $\begin{array}{l}\text { HgalC }<6,5 \% \text { antes } \\
\text { das } 13 \text { semanas: } \\
n(\%)\end{array}$ & $18(54,5)$ & $12(37,5)$ & $9(25)$ & $13(41,9)$ & 0,09 \\
\hline $\begin{array}{l}\text { HgalC }<6,5 \% \text { entre } \\
13-27 \text { semanas: } \\
n(\%)\end{array}$ & $24(70,6)$ & $28(75,7)$ & $19(52,8)$ & $27(67,5)$ & 0,2 \\
\hline $\begin{array}{l}\text { HgalC <6,5\% após } \\
27 \text { semanas: n (\%) }\end{array}$ & $27(64,3)$ & $21(63,6)$ & $19(57,6)$ & $20(54,1)$ & 0,7 \\
\hline
\end{tabular}

Tabela 3. Informações sobre a via de parto e peso dos recém-nascidos

\begin{tabular}{lccccc}
\hline & $\begin{array}{c}2004- \\
2005 \\
(n=46)\end{array}$ & $\begin{array}{c}2006- \\
2007 \\
(n=40)\end{array}$ & $\begin{array}{c}2008- \\
2009 \\
(n=42)\end{array}$ & $\begin{array}{c}2010- \\
2011 \\
(n=40)\end{array}$ & Valor p \\
\hline $\begin{array}{l}\text { IG* parto }>37 \\
\text { semanas }\end{array}$ & $27(58,7)$ & $26(65)$ & $27(64,3)$ & $32(80)$ & 0,1 \\
$\begin{array}{l}\text { Cesárea eletiva } \\
\text { RN* peso }>4000 \mathrm{gg}\end{array}$ & $24(52,2)$ & $17(42,5)$ & $30(71,4)$ & $18(45)$ & 0,03 \\
RN GIG* & $15(32,6)$ & $17(25)$ & $8(19)$ & $3(7,5)$ & 0,1 \\
\hline
\end{tabular}

*IG: Idade gestacional; RN: Recém-nascido; GIG: Grandes para a idade gestacional. 
ponderal na gravidez foi excessivo. Pela grande dispersão de dados e pelo reduzido tamanho amostral, optou-se por não estudar a variação do ganho ponderal dentro de cada categoria de IMC pré-gestacional ao longo dos anos de estudo.

Em $67 \%$ o parto foi de termo e, apesar de não ter significância estatística, observou-se um aumento progressivo dos partos de termo, tendo ocorrido em $80 \%$ dos casos em 2010-11. O parto por cesárea eletiva ocorreu em $53 \%(n=89)$, registrando-se uma redução significativa desse tipo de parto $(\mathrm{p}=0,03)$, ao longo dos anos de estudo.

Dados totais relativos aos motivos para cesárea eletiva relacionados com as complicações do DM foram: 25 casos por suspeita de macrossomia; 5 casos por retinopatia grave contraindicando o parto vaginal; 5 casos por más condições de indução do trabalho de parto (ITP) no contexto de pré-eclâmpsia grave; e 12 casos por cesárea anterior sem condições de oferecimento de prova de trabalho.

Observou-se uma tendência de redução na proporção de neonatos considerados grandes para o IG ao longo dos anos em estudo $(\mathrm{p}=0,039)$.

\section{Discussão}

no presente estudo foram apresentadas variações epidemiológicas, de perfil clínico e de resultados das gestações com diagnóstico de DM, seguidas em um único centro com apoio perinatal diferenciado de Portugal. Observou-se um aumento global na prevalência de diabetes preexistente à gravidez ${ }^{1,13,14}$. Esse aumento, para além de baseado no aumento da prevalência da doença em idades mais jovens ${ }^{15,16}$, reflete o aumento dos casos de DM tipo 2 demonstrado em estudos de base populacional, principalmente em países desenvolvidos ${ }^{8,13}$. Em nosso estudo, os resultados foram divergentes dessa realidade: globalmente, nenhuma tendência foi encontrada em relação ao número de gestantes com DM seguidas no nosso centro. No biênio 2010-2011 houve um decréscimo no número de casos referenciados em relação aos seguidos em igual período em 2008-2009, que coincidiu com a reorganização dos centros de referenciação de gestantes diabéticas na região central do país. Da mesma forma, em nosso estudo não se verificou nenhuma variação em relação à idade materna ou em relação à incidência dos casos de DM tipo2 ao longo dos anos. Todas essas variáveis permaneceram constantes ao longo do período de estudo.

Em relação à raça das gestantes os dados apresentados também não confirmam os encontrados na literatura, que apontam o aumento dos casos de DM tipo 2 em mulheres de raça não branca ${ }^{17-19}$. Em nossa amostra, apesar do ligeiro aumento de casos em gestantes de raça não branca nos últimos anos de estudo em relação aos primeiros, a maioria dos casos corresponderam a gestantes de raça branca.

Vários estudos apontam que o planejamento da gravidez em mulheres portadoras de DM tem um impacto significativo na redução de riscos materno-fetais ${ }^{4,9,20}$. Quando se avaliaram os cuidados pré-concepcionais oferecidos a essas mulheres, verificou-se que alguns parâmetros ficaram aquém do esperado, o fato de não se ter registrado nenhuma melhora na proporção de gestantes que tiveram consulta pré-concepcional foi desapontador. No entanto, a análise de resultados em relação aos cuidados de pré-natal precoces na gestação registraram progresso. A referenciação no primeiro trimestre da gestação e a consulta diferenciada e multidisciplinar, entre os anos de 2004 e 2009, registraram progressos muito significativos. Em igual período foi possível obter uma avaliação precoce do valor de $\mathrm{HbA1c}$ em proporção crescente nas gestantes seguidas.

$\mathrm{Na}$ avaliação clínica dessas mulheres, é também fundamental a observação do fundo ocular previamente à gravidez ou de forma precoce em seu decurso; o período gestacional constitui uma janela de oportunidade de avaliação, seguimento e terapêutica das complicações microvasculares da doença ${ }^{21}$. Em nosso centro os resultados foram excelentes ao longo de todo o período de estudo.

Outro fator importante para melhorar o prognóstico obstétrico de gestantes diabéticas é o controle do aumento de peso materno. A obesidade materna e o ganho de peso acima do recomendado estão associados aos piores resultados obstétricos ${ }^{22-24}$. $\mathrm{Na}$ avaliação do ganho ponderal das nossas gestantes, o qual foi aferido de acordo com o IMC prévio e ajustado à IG do parto, os resultados não demonstraram melhora ao longo dos anos de estudo. Em 39\% do total de gestantes o ganho ponderal foi excessivo. Esse dado levou à reflexão de que apesar de ter sido oferecido acompanhamento nutricional específico, as gestantes não tiveram uma boa adesão às orientações da equipe.

As complicações gestacionais/fetais classicamente associadas ao diabetes são as malformações congênitas, o aborto espontâneo e a macrossomia. A hiperglicemia é o deterninante mais importante desses riscos ${ }^{25-27}$. De acordo com a literatura, no presente estudo foram demonstrados valores de $\mathrm{HgAlC}$ média no $1^{\circ}$ trimestre superiores nas gestantes que tiveram abortos espontâneos. Em contraste com essa realidade, nas gestantes que não tiveram perda gestacional antes da $24^{\mathrm{a}}$ semana, os valores de controle metabólico foram globalmente muito promissores, apesar de não se ter demonstrado nenhuma variação ao longo do tempo. Grande proporção das gestantes atingiu baixos níveis de $\mathrm{HgA1}$ c no segundo trimestre e, apesar do aumento da resistência à insulina, próprio do terceiro trimestre, os resultados de bom controle metabólico mantiveram-se igualmente altos nesse trimestre.

A avaliação da proporção de neonatos GIG, no presente estudo, revelou melhoras significativas, registrando-se uma redução da proporção desses neonatos ao longo do tempo de estudo. Da mesma forma e em igual periodo, observou-se uma redução significativa de parto por cesárea eletiva. Esses resultados levaram à reflexão de que o bom controle metabólico foi, com certeza, decisivo. No entanto, é importante lembrar que nesses tipos de gestações, é certo que muitos outros fatores implicam o pior prognóstico dessas gestantes quando comparadas com gestantes de baixo risco. Evers e 
colaboradores publicaram dois importantes estudos prospectivos que demonstraram que gestantes com DM tipo 1 têm pior prognóstico obstétrico, mesmo em condições de ótimo ou subótimo controle metabólico ${ }^{28,29}$.

Apesar do exposto, continuamos acreditando que a avaliação pré-concepcional, a vigilância precoce e por equipe multidisciplinar, um bom controle metabólico antes e durante toda a gestação e a melhora no controle de aumento de peso inadequado por parte de gestantes portadoras de DM contribuem de forma decisiva para a melhora do prognóstico obstétrico dessas gestações de alto risco.

\section{Referências}

1. Lawrence JM, Contreras R, Chen W, Sacks DA. Trends in the prevalence of preexisting diabetes and gestational diabetes mellitus among a racially/ethnically diverse population of pregnant women, 1999-2005. Diabetes Care. 2008;31 (5):899-904.

2. Persson $M$, Norman $M$, Hanson U. Obstetric and perinatal outcomes in type 1 diabetic pregnancies: a large, population-based study. Diabetes Care. 2009;32(1 1):2005-9.

3. Peticca P, Keely EJ, Walker MC, Yang Q, Bottomley J. Pregnancy outcomes in diabetes subtypes: how do they compare? A province-based study of Ontario, 2005-2006. J Obstet Gynaecol Can. 2009;31 (6):487-96.

4. Pearson DW, Kernaghan D, Lee R, Penney GC; Scottish Diabetes in Pregnancy Study Group. The relationship between pre-pregnancy care and early pregnancy loss, major congenital anomaly or perinatal death in type I diabetes mellitus. BJOG. 2007;1 14(1):104-7.

5. Temple R, Aldridge V, Greenwood R, Heyburn P, Sampson M, Stanley $K$. Association between outcome of pregnancy and glycaemic control in early pregnancy in type 1 diabetes: population based study. BM. 2002;325(7375):1275-6.

6. Gonzalez-Gonzalez NL, Ramirez O, Mozas J, Melchor J, Armas $H$, Garcia-Hernandez JA, et al. Factors influencing pregnancy outcome in women with type 2 versus type 1 diabetes mellitus. Acta Obstet Gynecol Scand. 2008;87(1):43-9.

7. Bell R, Bailey K, Cresswell T, Hawthorne G, Critchley J, Lewis-Barned N, et al. Trends in prevalence and outcomes of pregnancy in women with preexisting type I and type II diabetes. BJOG. 2008; 1 15(4):445-52.

8. Yang J, Cummings EA, $\mathrm{O}^{\prime}$ connell $\mathrm{C}$, Jangaard $\mathrm{K}$. Fetal and neonatal outcomes of diabetic pregnancies. Obstet Gynecol. 2006;108(3 Pt 1):644-50.

9. Cyganek K, Hebda-Szydlo A, Katra B, Skupien J, Klupa T, Janas I, et al. Glycemic control and selected pregnancy outcomes in type 1 diabetes women on continuous subcutaneous insulin infusion and multiple daily injections: the significance of pregnancy planning. Diabetes Technol Ther. 2010;12(1):41-7.

10. Institute of Medicine; National Research Council Committee to Reexamine IOM Pregnancy Weight Guidelines; Rasmussen KM, Yaktine AL;. Weight gain during pregnancy: reexamining the guidelines. Washington (DC): National Academies Press; 2009.

11. Lapolla A, Dalfrà MG, Fedele D. Pregnancy complicated by diabetes: what is the best level of $\mathrm{HbAlc}$ for conception? Acta Diabetol. 2010;47(3):187-92.

12. Olsen IE, Groveman SA, Lawson ML, Clark RH, Zemel BS. New intrauterine growth curves based on United States data. Pediatrics. 2010;125(2):e214-24.

13. Feig DS, Razzaq A, Sykora K, Hux JE, Anderson GM. Trends in deliveries, prenatal care, and obstetrical complications in women with pregestational diabetes: a population-based study in Ontario, Canada, 1996-2001. Diabetes Care. 2006;29(2):232-5.

14. Feig DS, Palda VA. Type 2 diabetes in pregnancy: a growing concern. Lancet. 2002;359(9318):1690-2.
15. Liese AD, D’Agostino RB Jr, Hamman RF, Kilgo PD, Lawrence JM, Liu LL, et al. The burden of diabetes mellitus among US youth: prevalence estimates from the SEARCH for Diabetes in Youth Study. Pediatrics. 2006; 1 18(4):1510-8.

16. Pinhas-Hamiel $O$, Zeitler $P$. The global spread of type 2 diabetes mellitus in children and adolescents. J Pediatr. 2005;146(5):693-700.

17. Macintosh MC, Fleming KM, Bailey JA, Doyle P, Modder J, Acolet D, et al. Perinatal mortality and congenital anomalies in babies of women with type 1 or type 2 diabetes in England, Wales, and Northern Ireland: population based study. BM. 2006;333(7560):177-80.

18. Mayer-Davis EJ, Beyer J, Bell RA, Dabelea D, D'Agostino R Jr, Imperatore $G$, et al. Diabetes in African American youth: prevalence, incidence, and clinical characteristics: the SEARCH for Diabetes in Youth Study. Diabetes Care. 2009;32 (Suppl 2):S1 12-22.

19. Bell RA, Mayer-Davis EJ, Beyer JW, D'Agostino RB Jr, Lawrence JM, Linder $B$, et al. Diabetes in non-Hispanic white youth: prevalence, incidence, and clinical characteristics: the SEARCH for Diabetes In Youth Study. Diabetes Care. 2009;32 (Suppl 2):S102-11.

20. Cyganek K, Hebda-Szydlo A, Skupien J, Katra B, Janas I, Borodako $A$, et al. Glycemic control and pregnancy outcomes in women with type 2 diabetes from Poland. The impact of pregnancy planning and a comparison with type 1 diabetes subjects. Endocrine. 201 1;40(2):243-9.

21. American Diabetes Association. Executive summary: standards of medical care in diabetes - 2011 . Diabetes Care. 2011;34 (Suppl 1):S4-10.

22. Padilha PC, Saunders C, Machado RCM, Silva CL, Bull A, Sally $E O F$, et al. [Association between pre-gestational nutritional status and prediction of the risk of adverse pregnancy outcome]. Rev Bras Ginecol Obstet. 2007;29(10):51 1-8. Portuguese.

23. Roman H, Robillard PY, Hulsey TC, Laffitte A, Kouteich K, Marpeau $L$, et al. Obstetrical and neonatal outcomes in obese women. West Indian Med J. 2007;56(5):421-6.

24. Kristensen J, Vestergaard M, Wisborg K, Kesmodel U, Secher NJ. Pre-pregnancy weight and the risk of stillbirth and neonatal death. BJOG. 2005; $112(4): 403-8$.

25. Lindell G, Maršál K, Källén K. Impact of maternal characteristics on fetal growth in the third trimester of pregnancy. A population-based study. Ultrasound Obstet Gynecol. 2012 Feb 3. [Epub ahead of print].

26. Guerin A, Nisenbaum R, Ray JG. Use of maternal GHb concentration to estimate the risk of congenital anomalies in the offspring of women with prepregnancy diabetes. Diabetes Care. 2007;30(7):1920-5.

27. Moore TR, Jovanovic L. Pregnancy risks in women with type 1 and type 2 diabetes mellitus [Internet]. Up to date. 2012 [cited 2012 Ago 12]. Available from: <http://www.uptodate.com/contents/ pregnancy-risks-in-women-with-type-1-and-type-2-diabetes-mellitus>.

28. Evers IM, de Valk HW, Visser GH. Risk of complications of pregnancy in women with type 1 diabetes: nationwide prospective study in the Netherlands. BM. 2004;328(7445):915.

29. Evers IM, de Valk HW, Mol BW, ter Braak EW, Visser GH. Macrosomia despite good glycaemic control in Type I diabetic pregnancy; results of a nationwide study in The Netherlands. Diabetologia. 2002;45(1 1):1484-9. 